\title{
Caregivers' evaluation of an intervention program to improve executive functions of patients with mild neurocognitive disorder in Japan
}

\author{
Daisuke Fukuta* and Chizuru Mori \\ Faculty of Nursing, Tokiwa University, Japan
}

\begin{abstract}
Objective: The purpose of this study was to verify the feasibility of an intervention program designed to improve the executive functions of patients with mild neurocognitive disorder from the perspective of evaluation by caregivers in Japan.

Design: Non-randomized clinical trial

Methods: Participants were divided into the intervention group $(\mathrm{n}=10)$ and control group $(\mathrm{n}=10)$. The intervention group performed an intervention program based on Lezak's conceptual model that teaches task-specific routines. The program consisted of six training sessions presented over a two-week period. The Dysexecutive Questionnaire (DEX) by patients and caregivers were performed pre- and postintervention and once in the control group.

Results: The DEX-patient and DEX-caregiver total score was improved postintervention $(p<0.01)$. The DEX-caregiver total score was significantly higher than the DEX-patient total score at preintervention and that in the control group $(p<0.05)$, while there were no significant differences at postintervention.

Conclusion: We suggest that patients with mild neurocognitive disorder had low awareness of their own executive dysfunctions, while caregivers were able to definitely observe their own executive dysfunctions. The perspective of caregivers was important when evaluating detailed changes in executive functions because they were closely observing the real life of inpatients with mild neurocognitive disorder. This intervention program could be expected to improve the quality of life for not only patients with mild neurocognitive disorder but also their caregivers.
\end{abstract}

\section{Introduction}

Estimates of the prevalence of mild neurocognitive disorder, previously known as mild cognitive impairment, range from $2 \%$ to $10 \%$ at age 65 and $5 \%$ to $25 \%$ by age 85 [1]. The diagnostic criteria for mild neurocognitive disorder are one or more modest cognitive declines from a previous level of performance in abilities [1]. Patients with mild neurocognitive disorder require greater effort, compensatory strategies, or accommodation in terms of complex instrumental activities of daily living (instrumental ADL). In recent research on mild neurocognitive disorder, executive dysfunction was reported to be associated with declines in instrumental ADL [2,3]. Executive functions comprised four components: volition, planning, purposive action, and effective performance [4]. Previous studies reported that patients with mild neurocognitive disorder had impaired planning ability in specific aspects of executive functions $[2,5,6]$. In addition, patients with mild neurocognitive disorder had low awareness of their own executive dysfunctions, even though caregivers had definitely observed such executive dysfunctions in these patients. Experience of failure in everyday life leads to loss of self-confidence for patients with mild neurocognitive disorder and promotes anxiety of caregivers living with them [7]. Therefore, to reduce the anxiety of caregivers living with patients with mild neurocognitive disorder, an intervention program that allows caregivers to recognize improvement of executive functions for such patients will be necessary. Therefore, the purpose of this study was to verify the feasibility of an intervention program designed to improve executive functions of patients with mild neurocognitive disorder from the perspective of evaluation by caregivers in Japan.

\section{Materials and methods}

\section{Research design}

This study was a non-randomized clinical trial consisting of intervention group and non-intervention group (control group).

\section{Participants}

Twenty pairs of patients with mild neurocognitive disorder and their caregivers of a university hospital in Japan were recruited to participate in this study. The participants were divided into the intervention group (inpatients, $\mathrm{n}=10$ ) and control group (outpatients, $\mathrm{n}=10$ ) (Table 1). Inpatients were the participants who were diagnosed with mild neurocognitive disorder, hospitalized, and scheduled for discharge within a few weeks. Outpatients were the participants who were already living with their caregivers at home. The diagnosis of mild neurocognitive disorder was made based on the criteria of the fifth edition of the Diagnostic and Statistical Manual of Mental Disorders [1].

${ }^{\star}$ Correspondence to: Daisuke Fukuta, Faculty of Nursing, Tokiwa University, Japan, E-mail: dfukuta@tokiwa.ac.jp

Key words: caregiver, executive functions, intervention program, mild neurocognitive disorder, non-randomized clinical trial

Received: September 20, 2018; Accepted: October 03, 2018; Published: October 09,2018 
Table 1. Baseline data of study participants $(n=20)$

\begin{tabular}{|c|c|c|c|c|c|c|c|c|}
\hline \multirow[b]{2}{*}{ Patients } & \multicolumn{4}{|c|}{ Intervention group $(n=10)$} & \multicolumn{4}{|c|}{ Control group $(n=10)$} \\
\hline & Age & Sex & MMSE & Caregiver & Age & Sex & MMSE & Caregiver \\
\hline 1 & 76 & $\mathrm{~F}$ & 25 & Adult child & 75 & $\mathrm{~F}$ & 23 & Spouse \\
\hline 2 & 75 & $\mathrm{~F}$ & 24 & Spouse & 67 & $\mathrm{~F}$ & 22 & Spouse \\
\hline 3 & 70 & $\mathrm{~F}$ & 21 & Adult child & 76 & $\mathrm{~F}$ & 21 & Adult child \\
\hline 4 & 71 & $\mathrm{~F}$ & 30 & Spouse & 74 & $\mathrm{~F}$ & 26 & Adult child \\
\hline 5 & 71 & M & 29 & Spouse & 60 & M & 21 & Adult child \\
\hline 6 & 68 & $\mathrm{~F}$ & 29 & Spouse & 79 & $\mathrm{~F}$ & 25 & Spouse \\
\hline 7 & 62 & $\mathrm{~F}$ & 30 & Spouse & 67 & $\mathrm{~F}$ & 28 & Spouse \\
\hline 8 & 60 & $\mathrm{~F}$ & 25 & Spouse & 74 & M & 21 & Spouse \\
\hline 9 & 79 & $\mathrm{~F}$ & 23 & Adult child & 77 & $\mathrm{~F}$ & 27 & Adult child \\
\hline 10 & 62 & $\mathrm{~F}$ & 26 & Spouse & 67 & $\mathrm{~F}$ & 24 & Spouse \\
\hline
\end{tabular}

There were no statistically significant differences between the intervention group and control group in age and MMSE score with Mann-Whitney U test. MMSE, Mini-Mental State Examination

The inclusion criteria of patients were as follows: (1) age older than 60 years, (2) Mini-Mental State Examination (MMSE) score [8] $>20$, (3) Beck Depression Inventory (BDI) score [9] > 14, (4) absence of vascular lesions, and (5) absence of psychiatric disorder that could cause cognitive impairment.

\section{Intervention program to improve executive functions of patients with mild neurocognitive disorder}

The intervention program consisted of six sessions presented over a 2-week period, three times per week. Sessions lasted 30-60 min [10]. The first session's objective-to identify the task-focused on assessing the patient's current daily functioning and self-confidence for life after discharge and on selecting a task that would improve his or her daily functioning based on Lawton's instrumental ADL domains [11]. Examples of the tasks include the domains of shopping, food preparation, responsibility for own medications, and laundry. The importance of the first session was to empower the patient to use his or her awareness and internal motivation [12].

The second session's objective-formulation of a goal-focused on clearly defining and, then, writing down a goal for the task that would be achievable in 2 weeks. Clearly defining an achievable goal would lead to high motivation on the part of the participants. For example, the participants formulate a goal such as doing the shopping necessary for cooking, preparing, and serving adequate meal, taking medication in correct dosages at the correct time, or doing personal laundry completely.

The third session's objective-planning and sequencing-focused on writing a task analysis in which the routine was broken into single, logically sequenced steps, which were then simplified into three behaviors, namely, preparation, practice, and confirmation, with a place to check off each step as it was completed. Task analysis checklists served not only to plan, sequence, and solve complex problems and executive multistep procedures, but also to help reestablish everyday functioning [13]. As an example, food preparation can be simplified as follows: preparation, "plan the menu, and gather all necessary ingredients and cooking equipment"; practice, "cook a lunch for two and adjust the seasoning"; and confirmation, "turn off the gas or electric ovens."

The fourth session's objective-the characteristics of the executive functions-focused on understanding the definition of the executive functions and on error correction of the order for the function based on Lezak's conceptual model [4]. Errors produced during learning interfere with correct responses [14]. Baddeley and Wilson
[15] reported that errorless learning enhances learning in patients with amnesia and reduces forgetting rates. Several researchers have identified errorless learning as a useful rehabilitation tool in dementia [16-18]. This session helps patients understand the correct order of executive functions and improve efficiency in planning.

The fifth session's objective-practice-focused on sufficient practice for each step, consisting of two stages. In the first stage, the participant reads aloud the steps from the beginning that were developed in session 3. The practice of reading aloud is based on self-instruction training. Self-instruction training refers to the process of using self-talk messages or deliberately following specific task completion routines to regulate behavior and successfully complete goal-directed activities. Consistent practice and support for evaluating one's performance can result in improved error recognition and correction [19]. It is speculated that self-instruction training is useful in improving planning and problem-solving [20]. In the second stage of the practice, assuming the life after discharge, the participants performed sufficient training using real objects, such as cooking equipment, replica food, prescribed drug, or clothes. This session was performed twice.

The sixth session's objective-self-reflection-focused on reflection about the program with the researcher and on posttest.

\section{Instruments}

\section{Assessment of executive functions}

Dysexecutive questionnaire (DEX): The DEX is a valid questionnaire that assesses problems in the everyday behavior of patients with dysexecutive syndromes [21] (Table 2). Moreover, the DEX is included in a valid battery of Behavioral Assessment of Dysexecutive Syndrome. The DEX questionnaire comprises 20 items, with a 5-point Likert scale of 0 (never) to 4 (very often), with higher scores indicating more problems. Two versions are used: DEX self-rating performed by patients (DEX-patient) and DEX other-rating filled out by others who know the patient well both currently and premorbidly such as spouse, parents, siblings, and adult child (DEX-caregivers). The maximum total score is 80 points on each questionnaire. The usefulness of the DEX for mild cognitive impairment has been validated [5]. The DEX has been translated into Japanese [22], and the reliability and validity of the Japanese version of the DEX for mild neurocognitive disorder have been confirmed [2].

The DEX by patients and caregivers were performed pre- and postintervention in the intervention group and once in the control group. 
Table 2. Item score of DEX-caregiver $(n=20)$

\begin{tabular}{|c|c|c|c|c|c|c|c|c|}
\hline & \multirow{3}{*}{ Item } & \multicolumn{7}{|c|}{ Control group $(n=10)$} \\
\hline & & \multicolumn{2}{|c|}{ Preintervention } & \multicolumn{2}{|c|}{ Postintervention } & \multirow[b]{2}{*}{$p$} & \multirow[b]{2}{*}{$M$} & \multirow[b]{2}{*}{$S D$} \\
\hline & & $M$ & $S D$ & $M$ & $S D$ & & & \\
\hline 1. & Has problems understanding what other people mean unless they keep things simple and straightforward & 1.00 & 1.85 & 0.13 & 0.35 & & 0.90 & 0.88 \\
\hline 2. & Acts without thinking, doing the first thing that comes to mind & 0.50 & 1.41 & 0.25 & 0.71 & & 0.60 & 1.35 \\
\hline 3. & Sometimes talks about events or details that never actually happened, but she/he believes did happen & 0.50 & 1.41 & 0.00 & 0.00 & & 0.30 & 0.95 \\
\hline 4. & Has difficulty thinking ahead or planning for the future & 0.75 & 1.49 & 0.25 & 0.46 & & 0.70 & 1.49 \\
\hline 5. & Sometimes gets over-excited about things and can be a bit "over-the-top" at these times & 0.50 & 1.41 & 0.00 & 0.00 & & 0.00 & 0.00 \\
\hline 6. & Gets events mixed up with each other and gets confused about the correct order of events & 0.25 & 0.71 & 0.00 & 0.00 & & 1.00 & 1.70 \\
\hline 7. & Has difficulty realizing the extent of his/her problems and is unrealistic about the future & 0.25 & 0.71 & 0.25 & 0.71 & & 0.00 & 0.00 \\
\hline 8. & Seems lethargic or unenthusiastic about things & 2.38 & 2.00 & 0.75 & 0.71 & * & 1.60 & 1.51 \\
\hline 9. & Does or says embarrassing things when in the company of others & 1.25 & 1.83 & 0.75 & 1.39 & & 0.90 & 1.45 \\
\hline 10. & Really wants to do something one minute, but couldn't care less about it the next & 2.63 & 1.77 & 0.63 & 0.74 & * & 0.40 & 1.26 \\
\hline 11. & Has difficulty showing emotion & 0.88 & 1.64 & 0.13 & 0.35 & & 0.20 & 0.63 \\
\hline 12. & Loses his/her temper at the slightest thing & 1.00 & 1.85 & 0.38 & 0.74 & & 1.00 & 1.41 \\
\hline 13. & Seems unconcerned about how she/he should behave in certain situations & 1.00 & 1.85 & 0.13 & 0.35 & & 0.70 & 1.34 \\
\hline 14. & Finds it hard to stop repeating saying or doing things once started & 1.00 & 1.85 & 0.13 & 0.35 & & 1.50 & 1.51 \\
\hline 15. & Tends to be very restless and "can't sit still" for any length of time & 1.75 & 1.98 & 0.38 & 0.52 & & 0.70 & 1.06 \\
\hline 16. & Finds it difficult to stop doing something even if s/he knows she/he shouldn't & 0.38 & 1.06 & 0.00 & 0.00 & & 0.10 & 0.32 \\
\hline 17. & Will say one thing, but do something different & 0.50 & 1.41 & 0.00 & 0.00 & & 1.10 & 1.29 \\
\hline 18. & Finds it difficult to keep his/her mind on something, and is easily distracted & 2.00 & 2.14 & 0.38 & 0.52 & & 1.20 & 1.48 \\
\hline 19. & Has trouble making decisions, or deciding what she/he wants to do & 1.88 & 2.03 & 0.63 & 0.74 & & 1.60 & 1.78 \\
\hline 20. & Is unaware of, or unconcerned about, how others feel about his/her behavior & 0.75 & 1.49 & 0.13 & 0.35 & & 0.30 & 0.95 \\
\hline
\end{tabular}

There were no statistically significant differences between the postintervention and control group for each item score in the Mann-Whitney U test. DEX, Dysexecutive Questionnaire; M, mean; $\mathrm{SD}$, standard deviation. ${ }^{*} p<0.05$, Comparison of pre- and postintervention by Wilcoxon signed rank test

\section{Ethics considerations}

The ethics committee of the Faculty of Medicine of the University of Tsukuba approved this study. All the participants provided written informed consent before the initiation of any research procedures. To ensure that they had enough information before making this decision, the researcher explained the aims and procedures of the study to the participants before asking them to sign consent forms. Moreover, the participants were informed about the possibility of opting out of the study and that their anonymity would be guaranteed during the data analysis and reporting. The instruments used in this study are generally permitted to be used; therefore, we referenced the sources in consideration of copyright.

\section{Data collection and analysis}

The Wilcoxon signed rank test was used to compare the pre- and postintervention DEX results. The Mann-Whitney U test was used to compare the intervention and control group DEX results. Version 25 of the Statistical Package for Social Sciences (SPSS), at the 95\% confidence level, was used.

\section{Results}

\section{Description of the sample}

The mean (standard deviation (SD)) age of the inpatients of the intervention group was $69.4 \pm 6.4$ years, and their mean (SD) MMSE score was $26.2 \pm 3.2$ (Table 1). The mean (SD) age of the outpatients of the control group was $71.6 \pm 6.0$ years, and their mean (SD) MMSE score was $23.8 \pm 2.6$. There were no statistically significant differences between the intervention group and control group in age and MMSE score. Moreover, it was assumed that there was no difference in the intervention group and control group in the degrees of general cognitive function of patients with mild neurocognitive disorder. The patients in the intervention group selected a task from Lawton's instrumental ADL domains such as responsibility for own medications
( $\mathrm{n}=4)$, shopping $(\mathrm{n}=4)$, food preparation, and laundry $(\mathrm{n}=1$ each). Caregivers of the intervention group were spouse $(n=7)$ and adult child $(n=3)$ and those in the control group were spouse $(n=6)$ and adult child $(\mathrm{n}=4)$.

\section{Difference in the intervention group of DEX score}

The DEX-caregiver of two items was significantly improved at postintervention $(p<0.05$; Table 2$)$. The postintervention DEXpatient and DEX-caregiver total scores were significantly better than at preintervention $(p<0.01$; Table 3$)$. In the preintervention, the DEXcaregiver total scores (mean $=20.4, \mathrm{SD}=17.0)$ were significantly higher than DEX-patient total scores (mean $=9.4, \mathrm{SD}=8.6)(p<0.05)$, while there were no statistically significant differences at postintervention.

\section{Difference between the two groups of DEX score}

The postintervention DEX-caregiver total scores (mean $=5.7, \mathrm{SD}$ $=2.6$ ) were significantly lower than the control group scores (mean $=14.8, \mathrm{SD}=14.3)(p<0.05)$. There were no statistically significant differences between the postintervention and control group for each of the DEX-caregiver item score and DEX-patient total score. In the control group, the DEX-caregiver total score was higher than the DEXpatient total score $($ mean $=7.1, \mathrm{SD}=7.4)(p<0.05)$.

\section{Discussion}

The purpose of this study was to verify the feasibility of an intervention program designed to improve the executive functions of patients with mild neurocognitive disorder from the perspectives of evaluation by caregivers. One of the main findings in this study is that our results revealed that the DEX-caregiver total score at postintervention was significantly lower than that at preintervention. This means that caregivers were accurately observing improvement of patients' executive dysfunction during the intervention program. Moreover, DEX-caregiver items 8 and 10 were significantly better after intervention. We encouraged caregivers to participate in session 
Table 3. Differences between the two groups of DEX total score $(n=20)$

\begin{tabular}{|c|c|c|c|c|c|c|c|c|c|c|c|}
\hline & \multicolumn{7}{|c|}{ Intervention group $(n=10)$} & \multirow{2}{*}{\multicolumn{4}{|c|}{ Control group $(n=10)$}} \\
\hline & \multicolumn{3}{|c|}{ Preintervention } & \multicolumn{4}{|c|}{ Postintervention } & & & & \\
\hline & $M$ & $S D$ & (Range) & $M$ & $S D$ & (Range) & & $M$ & $S D$ & (Range) & $p$ \\
\hline DEX-patient & 9.40 & 8.57 & $(1-30)^{\dagger}$ & 4.40 & 4.19 & $(0-12)$ & 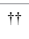 & 7.10 & 7.41 & $(0-20)^{\dagger}$ & \\
\hline DEX-caregiver & 20.40 & 16.99 & $(6-65)^{\dagger}$ & 5.70 & 2.58 & $(3-10)$ & 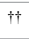 & 14.80 & 14.27 & $(3-52)^{\dagger}$ & * \\
\hline
\end{tabular}

DEX, Dysexecutive Questionnaire; M, mean; SD, standard deviation.

${ }^{*} p<.05$, comparison of postintervention and control group by Mann-Whitney U test

${ }^{\dagger} p<.05,{ }^{\dagger} \mathrm{p}<.01$, comparison of DEX-patient and DEX-caregiver and pre- and postintervention by Wilcoxon signed rank test

5 of an intervention program in which patients performed sufficient training using real objects when assuming the life after discharge. Consequently, caregivers would be able to recognize enhancement of enthusiasm, drive, and interest for patients' everyday activities from changes in their executive functions through the intervention. Moreover, it was assumed that caregivers' evaluation of executive dysfunction for patients led to declines in anxiety about their lives in the future. Interventions to reduce caregivers' evaluation were reported to negatively affect their caregiving ability for patients could be effective in enhancing their quality of life (QOL) [23]. Therefore, caregivers' objective evaluation of the executive functions of patients with mild neurocognitive disorder will be important.

Second, the DEX-caregiver total score was significantly higher than the DEX-patient total score at preintervention and control group. This indicates that patients with mild neurocognitive disorder had low awareness of their own executive dysfunctions, even if caregivers can definitely observe executive dysfunctions in such patients [2]. Some researchers have shown that patients with mild cognitive impairment lack awareness of their cognitive and behavioral deficits [24]. Moreover, lack of awareness of patients with mild neurocognitive disorder may be associated with executive dysfunction [25]. In the contrary, at postintervention, among patients and caregivers evaluated, there was no significant difference in DEX total score. It was thought that both patients and their caregivers were able to perceive that the patients' executive dysfunction had improved by an intervention program. In addition, the perspective of caregivers was important when evaluating changes in detailed executive functions because they were observing the real life of inpatients with mild neurocognitive disorder closely. Caregivers are in a unique and important position of being able to observe patients frequently. Therefore, it was inferred that caregivers have a role in the evaluation and support of patients with low awareness of their executive dysfunction and enhancement of patients' self-confidence every day.

Recently, there is a growing interest in rehabilitation for dementia from mild cognitive impairment to advance disease stage and functional decline [26]. In-home caregivers' positive evaluation for patients with dementia has affected the maintenance of caregiving [27]. Hence, intervention at the early stage of mild neurocognitive disorder and caregivers are required. Our findings suggested that the results of this study could verify the feasibility of an intervention program and would improve the QOL for not only patients with mild neurocognitive disorder but also their caregivers.

\section{Limitations}

Some limitations of the study should be taken into account with several suggestions for future research. First, given the small size and short-term intervention, the findings of this non-randomized clinical trial should be interpreted for only one clinical setting. Further studies are needed to validate these findings with a large sample size and data collection. Second, more studies are needed to clarify the long-term effectiveness of the intervention program by caregivers' evaluation with a follow-up period. Although this should be considered for future studies, our study has provided practical information on refining the intervention and research in the future.

\section{Implications for family health care nursing}

The research findings have proved that caregivers' evaluation for executive functions of patients with mild neurocognitive disorder was important to verify the usefulness and effectiveness of the intervention. This intervention program was developed to help patients with mild neurocognitive disorder improve planning ability, instrumental ADL, and self-confidence about everyday living with mild neurocognitive disorder [10]. We think it would be necessary for nurses to encourage caregivers to participate in the intervention program and to evaluate patients' executive dysfunction. Moreover, through the intervention, nurses would also need to support independent living at home of patients with mild neurocognitive disorder and to improve the QOL of patients and their caregivers.

\section{Conclusion}

Our findings suggest that caregivers were observing accurately improvement of patients' executive dysfunction through an intervention program. Caregivers could evaluate enhancement of enthusiasm, drive, and interest for patients' everyday activities. In addition, caregivers' evaluation of patients' executive dysfunction may reduce anxiety about patient's life in the future. Therefore, the perspective of caregivers was important when evaluating detailed changes in executive functions because they were observing closely the real life of inpatients with mild neurocognitive disorder. Further studies will be needed to verify the intervention to improve the QOL of patients with mild neurocognitive disorder living with their caregivers.

\section{Acknowledgements}

The authors are grateful to all the patients who participated in this research.

\section{References}

1. American Psychiatric Association (2013) Diagnostic and Statistical Manual of Mental Disorders (fifth edition) Text Revision. Arlington, VA: Author.

2. Fukuta D, Mori C (2018) Executive Dysfunction and Instrumental Activities of Daily Living in Mild Neurocognitive Disorder in Japan: A Preliminary Study. Int Med Journ 25: 86-89.

3. Marshall GA, Rentz DM, Frey MT, Locascio JJ, Johnson KA, et al. (2011) Executive function and instrumental activities of daily living in mild cognitive impairment and Alzheimer's disease. Alzheimers Dement 7: 300-308. [Crossref]

4. Lezak MD, Howison DB, Loring DW (2004) Neuropsychological assessment (4th ed.). New York, NY; Oxford University Press.

5. da Costa Armentano GC, Porto CS, Nitrini R, Dozzi Brucki SM (2013) Ecological Evaluation of Executive Functions in Mild Cognitive Impairment and Alzheimer Disease. Alzheimer Dis Assoc Disord 27: 95-101. [Crossref]

6. Espinosa A, Alegret M, Boada M (2009) Ecological assessment of executive functions in mild cognitive impairment and mild Alzheimer's disease. J Int Neuropsychol Soc 15: 751-757. [Crossref] 
7. Senba R, Kamijou K, Tabira T, Nishida S (2012) A literature review of dementia and instrumental activities of daily living. Japan Occupational Therapists Association 15: $7-12$

8. Folstein M, Folstein SE, McHugh PR (1975) "Mini-Mental State" a Practical Method for Grading the. Cognitive State of Patients for the Clinician. J Psychiatr Res 12: 189198. [Crossref]

9. Beck AT, Ward CH, Mendelson M, Mock J, Erbaugh J (1961) An inventory for measuring depression. Arch Gen Psychiatry 4: 561-571. [Crossref]

10. Fukuta D, Mori C (2018) Intervention program to improve executive functions and enhance planning abilities of patients with mild neurocognitive disorder. Rehabil Nurs [Crossref]

11. Lawton MP, Brody EM (1969) Assessment of older people: self-maintaining and instrumental activities of daily living. Gerontologist 9: 179-186. [Crossref]

12. Sohlberg MM, Mateer CA (1989) Introduction to cognitive rehabilitation: Theory and practice. New York, NY: Guilford Press.

13. Martelli M (2000) A Behavioral Protocol for Increasing Initiation and Activity. Rehabil Psychol News 27: 12-13.

14. Baddeley AD (1992) Implicit memory and errorless learning: A link between cognitive theory and neuropsychological rehabilitation? Neuropsychology of memory (2nd ed). New York, NY: Guilford Press.

15. Baddeley AD, Wilson BA (1994) When implicit learning fails: Amnesia and the problem of error elimination. Neuropsychologia 32: 53-68. [Crossref]

16. Mimura S, Sugano K (2005) Factors involved in learning efficacy of patients with mild cognition disorders. Psychiatria et neurologia Japonica 107: 1314-1319.

17. Clare L (2003) Cognitive training and cognitive rehabilitation for people with earlystage dementia. Reviews in Clinical Gerontology 5: 75-83. [Crossref]
18. Clare L, Wilson BA, Carter G, Roth I, Hodges JR (2004) Awareness in early-stage Alzheimer's disease: Relationship to outcome of cognitive rehabilitation. $J$ Clin Exp Neuropsychol 26: 215-226. [Crossref]

19. Sohlberg MM, Mateer CA (2001) Cognitive Rehabilitation: An Integrative Neuropsychological Approach. New York, NY: Guilford Press.

20. Meichenbaum DH, Goodman J (1971) Training impulsive children to talk to themselves: a means of developing self-control. J Abnorm Psychol 77: 115-126. [Crossref]

21. Wilson BA, Burgess P (1996) Behavioural Assessment of the Dysexecutive Syndrome: Test Manual. Bury St Edmonds, UK: Thames Valley Test Company.

22. Kashima H (2003) Behavioural Assessment of the Dysexecutive Syndrome (Jpn ed.) Tokyo, Japan; Shinkoh Igaku Shuppan.

23. Abdollahpour I, Nedjat S, Salimi Y, Noroozian M, Majdzadeh R (2015) Which variable is the strongest adjusted predictor of quality of life in caregivers of patients with dementia?. Psychogeriatrics 15: 51-57. [Crossref]

24. Rabin LA, Roth RM, Isquith PK, Wishart HA, Nutter-Upham KE, et al. (2006) Self- and informant reports of executive function on the BRIEF-A in MCI and older adults with cognitive complaints. Archives of Clinical Neuropsychology 21: 721-732. [Crossref]

25. Antoine P, Nandrino JL, Billiet C (2013) Awareness of deficits in Alzheimer's disease patients: analysis of performance prediction discrepancies. Psychiatry Clin Neurosci 67: 237-244. [Crossref]

26. Yamaguchi H, Maki Y, Yamagami T (2010) Overview of non-pharmacologica intervention for dementia and principles of brain-activating rehabilitation Psychogeriatrics 10: 206-213.

27. Kajiwara K, Nakatani H, Ono M, Miyakoshi Y (2015) Positive appraisal of in-home family caregivers of dementia patients as an influence on the continuation of caregiving. Psychogeriatrics 15: 26-31. [Crossref]

Copyright: $(02018$ Fukuta D. This is an open-access article distributed under the terms of the Creative Commons Attribution License, which permits unrestricted use, distribution, and reproduction in any medium, provided the original author and source are credited. 